Artículo Original

\title{
Asociación entre ser población vulnerable y el bajo nivel de conocimiento acerca de la COVID-19 en 17 ciudades del Perú al inicio de la pandemia
}

\section{Association between being a vulnerable population and low level of knowledge about COVID-19 in 17 cities of Peru at the beginning of the pandemic}

https://doi.org/10.52808/bmsa.7e5.61e2.007

Victor Serna-Alarcón ${ }^{1 *}$

https://orcid.org/0000-0002-9803-6217

Julio C Charri ${ }^{2}$

https://orcid.org/0000-0002-3613-3791

Humberto Garayar-Peceros ${ }^{3}$

https://orcid.org/0000-0003-4222-7230

Lizet Garay-Rios ${ }^{4}$

https://orcid.org/0000-0002-0577-7391

Macarena Carbajal ${ }^{5}$

https://orcid.org/0000-0003-1960-2953

Kevin Flores-Lovon ${ }^{6}$

https://orcid.org/0000-0001-6942-8118

J. Franco Rodriguez-Alarcón ${ }^{7,8}$

https://orcid.org/0000-0003-4059-8214

Lipselotte de Jesús Infante Rivera ${ }^{9}$ https://orcid.org/0000-0001-6094-1070

Martin A. Vilela-Estrada https://orcid.org/0000-0002-1494-952X

Christian R. Mejia ${ }^{10}$

https://orcid.org/0000-0002-5940-7281

\section{RESUMEN}

El conocimiento es pieza clave para afrontar cualquier enfermedad, pero se tiene pocos estudios basales de cuando empezó la pandemia del COVID19. El objetivo fue el determinar la asociación entre ser población vulnerable y el bajo conocimiento que tuvieron los pobladores en 17 ciudades del Perú al inicio de la pandemia. Se midió el conocimiento mediante una escala de nueve preguntas, que indaga acerca del mecanismo, los síntomas y las consecuencias; esto se cruzó versus variables socio-demográficas mediante la estadística analítica. De los 3913 encuestados, los menores conocimientos fueron acerca de cómo reaccionar ante síntomas de un resfrío y qué tratamiento debe seguir una persona que tiene infección inicial no grave por coronavirus ( $37 \%$ y $53 \%$ de respuestas correctas; respectivamente). En el análisis multivariado, los que tuvieron menores porcentajes de un mal nivel de conocimiento de la enfermedad fueron los que tenían estudios superiores (RPa: 0,74; IC95\%: 0,62-0,88; valor p=0,001), entre los que tenían algún post grado (RPa: 0,59; IC95\%: 0,43-0,68; valor p<0,001), entre los que eran parte del personal de salud (RPa: 0,15; IC95\%: 0,05-0,46; valor $\mathrm{p}=0,001$ ) y entre las mujeres (RPa: 0,84 ; IC95\%: 0,73-0,96; valor $\mathrm{p}=0,009$ ), en cambio, a mayor edad hubo un peor nivel de conocimientos de la enfermedad (RPa: 1,012; IC95\%: 1,005-1,018; valor p=0,001), ajustado por 4 variables. Se tuvo un bajo conocimiento en algunos aspectos y esto se asocia a algunas características específicas según ser considerado de población vulnerable.

Palabras clave: COVID-19, conocimientos, grupos de riesgo, Perú.

\section{ABSTRACT}

Knowledge is key to face any disease, but there are few baseline studies from the beginning of the COVID-19 pandemic. The objective was to determine the association between being a vulnerable population and the low knowledge of the population in 17 Peruvian cities at the beginning of the pandemic. Knowledge was measured using a nine-question scale, which asks about the mechanism, symptoms and consequences; this was crosschecked against socio-demographic variables using analytical statistics. Of the 3913 respondents, the lowest knowledge was about how to react to cold symptoms and what treatment a person with an initial non-severe coronavirus infection should follow (37\% and 53\% correct answers; respectively). In the multivariate analysis, those who had lower percentages of a poor level of knowledge of the disease were those with higher education (aPR: 0.74; 95\%CI: 0.62-0.88; p-value=0.001), among those with some postgraduate studies (aPR: 0.59; 95\%CI: 0.43-0.68; pvalue <0.001), among those who were part of the health personnel (aPR: 0.15; 95\%CI: 0.05-0.46; p-value=0.001) and among women (aPR: 0.84; 95\% CI: 0.73-0.96; p-value=0.009), on the other hand, the older the age, the worse the level of knowledge of the disease (aPR: 1.012; 95\%CI: 1.005- 
1.018; p-value=0.001), adjusted by 4 variables. There was a low level of knowledge in some aspects and this is associated with some specific characteristics according to being considered a vulnerable population.

Key words: COVID-19, knowledge, risk groups, Peru.

1. Escuela de Medicina Humana, Universidad Privada Antenor Orrego. Trujillo, Perú

2. Universidad Nacional Daniel Alcides Carrión. Cerro de Pasco, Perú.

3. Universidad Nacional San Luis Gonzaga de Ica. Ica, Perú.

4. Universidad Nacional del Centro del Perú. Huancayo, Perú.

5. Universidad Nacional Hermilio Valdizán. Huánuco, Perú.

6. Universidad Nacional de San Agustín de Arequipa. Arequipa, Perú.

7. Asociación Médica de Investigación y Servicios en Salud. Lima, Perú.

8. Facultad de Medicina Humana. Universidad Ricardo Palma. Lima, Perú. 9. Universidad Continental. Lima, Perú.

10. Centro de Investigación en Medicina Traslacional. Universidad Norbert Wiener. Lima, Perú.

\section{Introducción}

*Autor de Correspondencia: vsernaa@upao.edu.pe

La infección por SARS-CoV-2 se consolidó como la pandemia más importante del mundo, el 30 de enero del 2020 la OMS declaró en emergencia la salud pública por el brote chino de la COVID-19 (Sohrabi et al., 2020), la cual se inició a fines del año 2019 en las proximidades del mercado mayorista de mariscos en Huanan-Hubei (Chan et al., 2020; Zhu et al., 2020). Desde entonces se ha expandido a la gran mayoría de países en todo el globo terráqueo, causando millones de muertos, muchísimos más infectados, el colapso de diversas economías, entre otras muchas repercusiones (Chicaíza et al., 2021).

Esta enfermedad tiene características patológicas que permiten que se la confunda con un resfrío, siendo las principales manifestaciones clínicas la fiebre, la tos seca y la disnea (Wu et al., 2020 a; Zhou et al., 2020), esto por el hecho de que el 2019-nCoV produce este tipo de sintomatología (Zhou et al., 2020; Zhu et al., 2020). Además, se sabe que las personas con enfermedades crónicas, como hipertensión, diabetes, enfermedad renal crónica, entre otras, tienen mayor predisposición a presentar complicaciones durante el curso de la infección, requiriendo muchas veces ser intubados e internados en las Unidades de Cuidados Intensivos (UCI) (Baradaran et al., 2020; Qiu et al., 2020). De la misma manera, las mujeres embarazadas y otros grupos de riesgo, debido a los cambios fisiológicos, se encuentran más vulnerables a los patógenos respiratorios (Chen et al., 2020).

Por lo que, es importante saber reconocer que características tiene esta enfermedad en específico, la transmisión, los síntomas y hasta la tasa de mortalidad; sabiendo que mucha de la información se difundió por los medios de comunicación y las redes sociales (Mungmungpuntipantip \& Wiwanitkit, 2021), y en algunos casos la información es brindada por especialistas en el tema o instituciones acreditadas (Beldarraín Chaple, 2020). Pero fue tan diversa la fuente de información, que hasta a veces podría confundir a los que la reciben; además que al no saber del tema podría tomar malas decisiones y hasta entrar en pánico; sobre todo cuando esto se producen en una población no especializada (García-Saisó et al., 2021). Es por eso que el objetivo del estudio fue el determinar la asociación entre ser población vulnerable y el bajo conocimiento que tuvieron los pobladores en 17 ciudades del Perú al inicio de la pandemia.

\section{Materiales y Métodos}

La investigación tuvo un diseño de tipo transversal analítico y multicéntrico. Realizado en 17 ciudades del Perú. En cada ciudad se tuvo encuestados (en la medida de lo posible se guardó semejanza con el tamaño poblacional de cada departamento). Se incluyó a los que hayan mostrado interés por participar en la investigación y que tengan o no riesgo para una complicación por COVID-19 (ser anciano, paciente oncológico o con alguna enfermedad hipertensiva). Se excluyó a 63 personas por que residían en el extranjero al momento de la encuesta, 5 personas que respondieron de forma incongruente y 19 que no completaron varias preguntas del test. Se calculó que se requería a 3867 personas, esto para encontrar una diferencia mínima teórica de 2,5\% (48,5\% versus 51,0\%), para una potencia de 87,5\%, un nivel de confianza del $95 \%$ y para una muestra única. La muestra se tomó de forma no aleatoria, tratando de preservar la homogeneidad entre cada una de las sedes. Por lo que, las personas que ingresaron al estudio fueron un total de 3919.

El instrumento de investigación para la recolección de datos mide los conocimientos del mecanismo de infección, síntomas y consecuencias por el COVID-19, esto a través, de nueve preguntas, donde todos los encuestados tenían la posibilidad de marcar 1 de las 4 opciones de respuesta específicas para cada pregunta, a su vez todos los autores corroboraron que no haya errores en la clasificación de la respuesta correcta en cada pregunta. En el proceso para la elaboración de la encuesta fue de la siguiente manera: Una búsqueda primaria de artículos científicos en las bases más grandes del mundo, es aquí que se generó el primer borrador, luego se consultó la opinión de 30 expertos (infectólogos, internistas, intensivistas, epidemiólogos, entre otros). Posteriormente, se sometió a una comprobación estadística, en donde se evaluó la evidencia de validez y la cuantificación de la relevancia (en casi 400 encuestados a 
nivel nacional); en este paso se observó una buena representatividad y claridad. Por último, se realizó una validación estadística y la respectiva publicación (Mejia et al., 2020).

La recolección de algunas variables importantes fue considerada para poder generar la estadística analítica, como son: el sexo (masculino o femenino), la edad (en años cumplidos), el grado de instrucción (que para la estadística generaría dos variables: los que tienen hasta estudios superiores y los que hayan hecho un posgrado), el tipo de encuestado (

Se confeccionó un test virtual en Google Forms, luego del cierre de la encuesta se procedió a un control de calidad de los datos, en este paso se retiró a los que no cumplían los criterios de selección. Posteriormente se codificó y pasó los datos a una hoja en el programa Microsoft Excel (para Windows 2019). Una vez en la hoja Excel se realizó un segundo filtro y depuración de datos, así como, un control de la calidad por cada sede. Por último, se exportó la data al programa estadístico Stata (versión 11,1).

Para el análisis de datos se procedió a describir dichos ítems mediante sus frecuencias/porcentajes (variables categóricas) o las medianas/rangos intercuartílicos (variables cuantitativas). Posterior al análisis bivariado (se usó la prueba chi cuadrado, por cumplir el supuesto de normalidad, a través del supuesto de los valores esperados), se realizó el análisis multivariado. Para la obtención de las razones de prevalencia ajustadas, los intervalos de confianza al $95 \%$ y los valores p se hizo uso de la prueba de los modelos lineales generalizados; esto con familia de Poisson, la función de enlace log, modelos para varianzas robustas y con ajuste por cada una de las sedes de encuestado (esto por comprender que cada población es particular en sus costumbres, ámbitos sociales y hasta forma de percibir y reaccionar ante la enfermedad). Para considerar como estadísticamente significativos a los valores p se tomó como punto de corte al 0,05 .

En todo momento se respetó los parámetros éticos en la investigación. El proyecto fue presentado a un Comité de Ética en el Perú (aprobado por el comité de ética de la Universidad Privada Antenor Orrego), además, las encuestas fueron anónimas (para respetar la privacidad de los encuestados y para darles certeza de que no podríamos identificarlos; para que puedan responder con libertad acerca de sus percepciones).

\section{Resultados}

De los 3913 encuestados, el 55,2\% (2148) fueron mujeres, la mediana de edades fue de 23 años (rango intercuartílico: 20-28 años), el 81,5\% (3160) tenía estudios superiores, el 22,4\% (864) era personal de salud, el 6,6\% (255) tenían algún riesgo de complicación por COVID-19 (Tabla 1).

Tabla 1. Caracterización de la población en 17 ciudades del Perú

\begin{tabular}{|c|c|}
\hline Variables & $\begin{array}{c}\begin{array}{c}\text { Frecuencia } \\
(\%)\end{array} \\
\end{array}$ \\
\hline Sexo femenino & $2148(55,2 \%)$ \\
\hline Edad (años)* & $23(20-28)$ \\
\hline Es peruano & $3862(99,4 \%)$ \\
\hline Con estudios superiores & $3160(81,5 \%)$ \\
\hline Tiene posgrado & $299(7,7 \%)$ \\
\hline Es personal de salud & $864(22,4 \%)$ \\
\hline No tiene riesgo & $2810(72,9 \%)$ \\
\hline $\begin{array}{l}\text { Con riesgo de } \\
\text { complicación }\end{array}$ & $255(6,6 \%)$ \\
\hline
\end{tabular}

Las preguntas que eran más conocidas fueron las relacionadas a los síntomas de la enfermedad; tanto de los síntomas generales $(98,1 \%)$, como de los síntomas en específico $(94,3 \%)$. Además, de en quién es más alta la mortalidad de la enfermedad $(96,7 \%)$ y cuál es el mecanismo de transmisión de la enfermedad (94,3\%). En cambio, el menor conocimiento fue cómo reaccionar ante el tener síntomas y sospecha de infección por coronavirus (solo 36,7\% tuvo una respuesta correcta), así como, el que tratamiento se le debe dar a una persona que tiene una infección inicial no grave (solo 53,1\% tuvo una respuesta correcta) (Tabla 2).

Según las respuestas totales que tuvo cada encuestado, el 16,9\% de los encuestados tuvo 5 o menos respuestas correctas, además, el $38,4 \%$ tuvo 8 o 9 respuestas correctas $(23,7 \%$ y $14,7 \%$ con 8 y 9 respuestas, respectivamente). La mediana de respuestas correctas fue 7 (rango intercuartílico: 6-8 respuestas correctas). La figura 1 nos muestra el gráfico de cajas y bigotes de los puntajes de conocimiento de la enfermedad por coronavirus según el tipo de encuestado, en donde se puede apreciar que el grupo con mejores respuestas fue el que tenía algún riesgo para complicación y que era personal de salud, seguido por el personal de salud sin riesgo y las personas que no eran personal de salud y que tampoco tenían riesgo de complicaciones (Figura 1). 
Tabla 2. Conocimiento acerca de los síntomas, la mortalidad y cómo actuar en la pandemia de la COVID-19

\begin{tabular}{lc}
\hline \multicolumn{1}{c}{ Variables } & $\begin{array}{c}\text { Respuestas } \\
\text { correctas n }(\%)\end{array}$ \\
\hline $\begin{array}{l}\text { ¿Cuáles son los síntomas comunes que puede presentar una persona que tiene la infección } \\
\text { coronavirus? }\end{array}$ & $3840(98,1 \%)$ \\
De las siguientes alternativas ¿En quiénes es más alta la tasa de mortalidad del coronavirus? & $3783(96,7 \%)$ \\
¿Cuál de los siguientes NO es uno de los síntomas más comunes de la infección por coronavirus? & $3704(94,7 \%)$ \\
¿Cómo se transmite o cuál es el mecanismo de transmisión del coronavirus? & $3689(94,3 \%)$ \\
¿Cuál es el método diagnóstico que se utiliza para poder confirmar una infección por coronavirus? & $3173(81,1 \%)$ \\
¿Cuánto es el tiempo de incubación o en qué tiempo se pueden manifestar los síntomas del coronavirus? & $2865(73,2 \%)$ \\
¿Cuál es la probabilidad de morir (porcentaje de mortalidad) por coronavirus en la población general? & $2458(62,8 \%)$ \\
¿Qué tratamiento se le debe dar a una persona que tiene infección inicial (no grave) por coronavirus? & $2078(53,1 \%)$ \\
¿Qué harías si tienes síntomas de un resfrío y sospechas que estás infectado por coronavirus? & $1434(36,7 \%)$ \\
\hline Nota: Las preguntas no tenían esa aparición en el test, se han ordenado de mayor a menor cantidad de respuestas correctas.
\end{tabular}

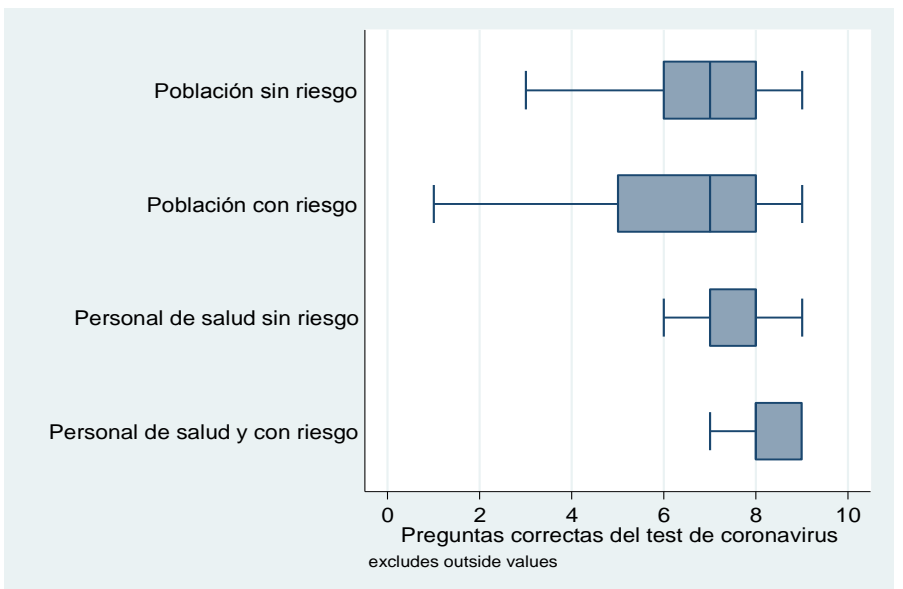

\section{Figura 1. Puntajes de conocimiento de la COVID-19 según la vulnerabilidad de población en Perú}

Según los porcentajes de errores que tuvieron los distintos grupos según cada una de las preguntas de conocimiento, la población en riesgo para una complicación fue la que tuvo los porcentajes más alto de errores, sobre todo en las preguntas del período de incubación (29\% de falla), síntomas generales (3\%), síntomas específicos (16\%), mortalidad general (46\%), mortalidad específica según grupos (11\%), tratamiento $(57 \%)$ y diagnóstico (22\%); casi todos los porcentajes fueron estadísticamente diferentes (en 8 casos se tuvo una diferencia <0,001); excepto en la

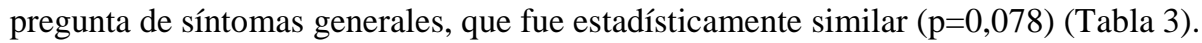

Cuando se obtuvo el análisis multivariado, se encontró que los que tuvieron menores porcentajes de un mal nivel de conocimiento de la enfermedad fueron los que tenían estudios superiores (RPa: 0,74; IC95\%: 0,62-0,88; valor $\mathrm{p}=0,001$ ), entre los que tenían algún post grado (RPa: 0,59; IC95\%: 0,43-0,68; valor $\mathrm{p}<0,001$ ), entre los que eran parte del personal de salud (RPa: 0,15; IC95\%: 0,05-0,46; valor $\mathrm{p}=0,001$ ) y entre las mujeres (RPa: 0,84; IC95\%: 0,73-0,96; valor $\mathrm{p}=0,009$ ), en cambio, a mayor edad hubo un peor nivel de conocimientos de la enfermedad (RPa: 1,012; IC95\%: 1,005-1,018; valor $\mathrm{p}=0,001)$, todo esto estuvo ajustado por los encuestados que si tenían algún riesgo de complicación, los que no tenían riesgo alguno, el ser peruano y el departamento donde residían (Tabla 4).

Tabla 3. Porcentaje de respuestas incorrectas por grupo poblacional en población peruana

\begin{tabular}{lccccc}
\hline \multicolumn{1}{c}{ Pregunta } & $\begin{array}{c}\text { Sin riesgo } \\
\mathbf{n = 2 7 5 5}\end{array}$ & $\begin{array}{c}\text { Con riesgo } \\
\mathbf{n = 2 3 8}\end{array}$ & $\begin{array}{c}\text { Del sector salud } \\
\mathbf{n = 8 4 7}\end{array}$ & $\begin{array}{c}\text { Sector salud y con riesgo } \\
\mathbf{n = 1 7}\end{array}$ & Valor p \\
\hline Transmisión & $7 \%$ & $6 \%$ & $2 \%$ & $12 \%$ & $<0,001$ \\
Incubación & $28 \%$ & $29 \%$ & $21 \%$ & $0 \%$ & $<0,001$ \\
Síntomas generales & $2 \%$ & $3 \%$ & $1 \%$ & $0 \%$ & 0,078 \\
Síntomas específicos & $5 \%$ & $16 \%$ & $2 \%$ & $6 \%$ & $<0,001$ \\
Mortalidad general & $40 \%$ & $46 \%$ & $27 \%$ & $12 \%$ & $<0,001$ \\
Mortalidad específica & $3 \%$ & $11 \%$ & $3 \%$ & $0 \%$ & $<0,001$ \\
Tratamiento & $53 \%$ & $57 \%$ & $26 \%$ & $12 \%$ & $<0,001$ \\
Diagnóstico & $21 \%$ & $22 \%$ & $12 \%$ & $12 \%$ & $<0,001$ \\
Caso sospechoso & $66 \%$ & $65 \%$ & $53 \%$ & $35 \%$ & $<0,001$ \\
\hline
\end{tabular}

Los valores $\mathrm{p}$ fueron calculados con la prueba chi cuadrado. 
Tabla 4. Análisis multivariado de las características asociadas a un mal conocimiento del coronavirus en Perú

\begin{tabular}{lccc}
\hline \multicolumn{1}{c}{ Variables } & Razón de prevalencia & Intervalos de confianza al 95\% & Valor p \\
\hline Con estudios superiores & 0,74 & $0,62-0,88$ & 0,001 \\
Tiene posgrado & 0,59 & $0,43-0,68$ & $<0,001$ \\
Es personal de salud & 0,15 & $0,05-0,46$ & 0,001 \\
No tiene riesgo & 0,43 & $0,13-1,36$ (No significativo) & 0,151 \\
Con riesgo de complicación & 0,52 & $0,15-1,77$ (No significativo) & 0,297 \\
Sexo femenino & 0,84 & $0,73-0,96$ & 0,009 \\
Edad (años)* & 1,012 & $1,005-1,018$ & 0,001 \\
Es peruano & 1,63 & $0,47-5,69$ (No significativo) & 0,445 \\
\hline
\end{tabular}

* La variable edad se tomó en su forma original (en años cumplido). Las razones de prevalencia ajustadas, los intervalos de confianza al $95 \%$ y valores p se obtuvieron con los modelos lineales generalizados, con la familia Poisson, función de enlace log, modelos robustos y ajustando por el departamento de residencia.

\section{Discusión}

Se encontró que los encuestados tenían un mejor nivel de conocimientos acerca de los síntomas, la mortalidad y el mecanismo de transmisión de la COVID-19. Esto es similar a lo reportado por un estudio donde la mayoría de los participantes reconoció la fiebre, la tos y la dificultad respiratoria como los principales síntomas de esta enfermedad, además el 93\% reconocía que la principal forma de contagio era por las gotitas respiratorias (ColoméHidalgo et al., 2021). De igual manera, en un estudio realizado en la capital del Perú, más de la mitad de los participantes conocía al menos un signo de alarma de esta enfermedad, y dentro de los síntomas que más identificaron se encontraban la fiebre, la cefalea, el dolor de garganta y la pérdida de gusto (Raraz Vidal et al., 2021). Probablemente estos resultados se deban a la gran campaña que se hizo para difundir esta información, además, de su gran parecido con los síntomas de un resfrío común, pues según refieren algunos estudios, los síntomas más comunes al inicio de la enfermedad son fiebre, fatiga, tos seca, mialgias y disnea (Gil et al., 2021; Struyf et al., 2021). Sin embargo, un estudio realizado en la sierra central del Perú encontró que el $81 \%$ de los encuestados percibía que desconocía el concepto de la COVID-19, y solo el $43 \%$ conocía cuáles eran los factores de riesgo y las medidas de prevención (Ruiz Aquino et al., 2021). Entonces, queda en evidencia que, si bien una gran parte de la población peruana tiene un adecuado nivel de conocimiento para identificar los síntomas y características más comunes de esta nueva enfermedad, aún hay sectores a los que no se llegando la información de manera adecuada.

En este estudio también se encontró un bajo nivel de conocimiento acerca de cómo actuar si es que una persona tiene síntomas y sospecha que tenga COVID-19, así como, qué tratamiento se le debe dar a una persona que tiene una infección inicial no grave por SARS-CoV-2, ya que, la mayoría de encuestados respondió que acudirían a un centro de salud y que buscarían internarse. Estos resultados difieren de un estudio realizado en República Dominicana, donde al preguntársele a los participantes acerca de qué se debería hacer en caso de tener una sospecha de COVID-19 con síntomas leves como tos o fiebre, el $76 \%$ respondió que la mejor opción era quedarse en casa y llamar a su médico de cabecera (Colomé-Hidalgo et al., 2021). Lo que muestra que en Perú de repente no se estuvo comunicando adecuadamente que el internamiento es solo para los casos graves, es decir, pacientes que presenten signos clínicos o radiológicos compatibles con neumonía, sepsis o insuficiencia respiratoria aguda (Lozano \& Palacios, 2021), pero para el manejo de pacientes con síntomas leves, se recomienda el aislamiento domiciliario y el uso de las medidas higiénicas; para no contagiar a los cuidadores y familiares, como el uso de mascarillas, el lavado de manos con agua y jabón o el uso de desinfectantes a base de alcohol (Ticona Huaroto et al., 2020). Entonces, esto debe evaluarse a profundidad y debe hacerse nuevos estudios, posterior a un gran periodo de tiempo (ya que, estos resultados muestran cómo se estaba en los primeros meses de la pandemia).

Aquellos que tenían estudios superiores, posgrado o los profesionales fueron quienes tuvieron un adecuado conocimiento de la enfermedad. Esto se esperaba, pues esta población tiene un mejor nivel de conocimiento por sus características académicas, sobre todo por el hecho de cómo consigue y asimila la información. En una encuesta aplicada a estudiantes universitarios, se encontró que más del $90 \%$ de ellos tenían conocimientos acerca de los síntomas de la COVID-19, además, casi la totalidad se encontraba de acuerdo con las medidas de prevención, como el lavado de manos y el uso de mascarillas (Alzoubi et al., 2020). Caso contrario ocurre con la población con menor educación, Akalu et al (2020) menciona que los pacientes que no saben leer ni escribir, tienen mayor probabilidad de tener un bajo nivel de conocimiento con respecto a aquellos que tienen un nivel educativo de secundaria o superior; y esto se ha evidenciado en varios estudios, en los que tener un menor nivel educativo estuvo asociado a un menor nivel de conocimiento acerca de la COVID-19 y, por lo tanto, una actitud menos favorable a cumplir con las medidas de prevención (Abdelhafiz et al., 2020; Ferdous et al., 2020; Qutob \& Awartani, 2021). Entonces es importante que el gobierno tome en cuenta esta información y se realicen campañas especiales que permitan llegar a esta población.

También se encontró que, el personal de salud, en especial quienes tenían algún factor de riesgo para desarrollar una complicación, fueron quienes tuvieron mejores respuestas. Esto también se ha visto en otro estudio, donde el 78\% del personal de salud encuestado reconocía correctamente la principal forma de transmisión del virus, así 
como, los síntomas iniciales predominantes de la infección (Guzmán et al., 2020). Además, Roth et al., (2021) encontraron que, si bien al inicio de la pandemia al personal de salud se le complicó un poco conseguir información sobre esta nueva enfermedad, durante la pandemia en curso y con el desarrollo de nuevas investigaciones, la mayoría de los encuestados consideraba que su nivel de conocimientos estaba actualizado. Si bien existen reportes de personal de salud afectado por esta patología, además, de ser un grupo con alta vulnerabilidad (Galán-Rodas et al., 2020) los factores de riesgo predisponentes reportados son el trabajar en áreas hospitalarias con amplia producción/flujo de aerosoles y una inadecuada higiene de manos (Ran et al., 2020). Por lo que, este grupo ocupacional debe estar vigilado, no solo por el hecho que debe conocer para poder tratar a los pacientes, sino también, por que debe tener mucho cuidado para conservar las medidas de protección cuando regrese al hogar; para que no infecte a su familia y amigos.

Sin embargo, en la población en general, fueron aquellas personas que tenían factores de riesgo quienes tuvieron un menor conocimiento. Esto resulta relevante, pues autores como Sánchez Ortiz (2016), mencionan que los pacientes con mejor conocimiento de su enfermedad tienen un mejor control de esta. Sabiendo que las principales comorbilidades de los casos de mortalidad de COVID-19 incluyen hipertensión, diabetes, enfermedad coronaria, infarto cerebral y bronquitis crónica (Deng \& Peng, 2020). Sin embargo, en un estudio realizado en Etiopía, en pacientes con enfermedades crónicas, solo el 37\% tenía un buen nivel de conocimiento acerca de la infección por SARS-CoV-2 (Akalu et al., 2020). Por otro lado, un estudio realizado en Vietnam encontró que el 63\% de participantes con enfermedades de larga data reconocía que, los pacientes con enfermedades crónicas subyacentes tenían un mayor riesgo de enfermar gravemente de COVID-19 y morir; además, la gran mayoría de ellos tenía un buen nivel de conocimiento acerca de las vías de transmisión del virus y de los principales síntomas (Huynh et al., 2020). Estas diferencias probablemente se deban a la diferencia de las campañas de difusión de información, ya que, en Perú estas no estuvieron articuladas con la estrategia sanitarias y fueron principalmente centralistas (Macassi, 2020).

Otro hallazgo de este estudio fue que, a mayor edad hubo un peor nivel de conocimiento. Esto también se ha visto en otro estudio, donde antes de realizarse una intervención educativa acerca de la COVID-19 a personas con edades entre 70 y 79 años, el $40 \%$ de ellos mostraba un nivel de conocimiento inadecuado, tanto de las medidas preventivas como de los signos y síntomas de esta nueva enfermedad (Díaz-Rodríguez et al., 2020). De igual manera, otro estudio realizado en una población de 65-70 años, evidenció que solo el 14\% de los participantes mostraba un nivel de conocimiento adecuado (Vázquez Chacón et al., 2019). Esta situación resulta preocupante, pues varios estudios han reportado que existe un mayor riesgo de complicaciones y progresión a la muerte en los adultos mayores (Ruiz Aquino et al., 2021; Wu et al., 2020 b); entonces, al ser una población de riesgo, resulta importante que se realice una mayor difusión de la información orientada hacia esta población, sobre todo en los temas de prevención.

Por último, fueron las mujeres quienes tuvieron un mejor nivel de conocimiento en comparación con los hombres. Resultados similares fueron encontrados en otros estudios, donde las mujeres no solo mostraban un mejor nivel de conocimiento acerca de la COVID-19, sino que tenían mejores prácticas de prevención (Rivera Diaz et al., 2020). Además, Levkovich \& Shinan-Altman (2021) mencionan que el comportamiento preventivo de las mujeres estaría asociado al nivel de conocimiento de la enfermedad y a presentar mayores reacciones emocionales. Estos resultados también se han visto en otras pandemias, como la del AH1N1, en la que si bien fueron los hombres quienes percibían mayores factores de riesgo para el contagio, fueron las mujeres las que tuvieron mejores medidas de prevención, así como, un mayor nivel de conocimiento de la enfermedad (Carro Pérez \& Hernández Gómez, 2016). Esto es importante, ya que, se ha reportado en múltiples ocasiones que son los hombres quienes tienen una mayor tasa de incidencia, mortalidad y letalidad por la COVID-19 (Falahi \& Kenarkoohi, 2020; Jin et al., 2020; Ramírez-Soto et al., 2021), y esto se puede deber a que, al cumplir con menos medidas de prevención, se encuentran más expuesto a la infección del virus.

El estudio presenta como limitación el sesgo de selección, ya que, al no tener una muestra aleatoria no se puede concluir que los resultados se apliquen a toda la población peruana. Sin embargo, al tener encuestados de todos los departamentos, así como, una muestra de casi 4000 encuestados, los autores consideran que esto se podría asemejar a la realidad. Cabe resaltar que es importante realizar estudios en la población rural, ya que, nuestra población es eminentemente urbana y que se encuestó durante las primeras semanas de la pandemia, así mismo, se deben hacer estudios situacionales por cada sub población específica (que ya se sabe muchísimo más de la infección, por lo que, los conocimientos deben haber mejorado).

Por todo lo mencionado, se concluye que algunos grupos en riesgo tienen conocimientos diferenciados; los que tienen un riesgo elevado para complicarse por COVID-19 tienen menor conocimiento y los que son del sector salud y tienen una patología de riesgo tienen un mayor conocimiento. Además, el nivel de conocimiento promedio es bueno, el grupo que más conocimiento tiene es el personal de salud que tiene riesgo de alguna complicación, sin embargo, el que menos conocimiento tiene es la población general con algún riesgo para complicación. Esto también estuvo asociado al nivel de instrucción, el grupo poblacional encuestado, la edad y el sexo. 


\section{Conflicto de intereses}

Los autores declaran no tener conflicto de interés.

\section{Agradecimientos}

A todos los miembros de la red COVID-19-GIS-Peru, que gracias a su invaluable apoyo se pudo obtener la data que generó esta investigación.

\section{Referencias}

Abdelhafiz, A. S., Mohammed, Z., Ibrahim, M. E., Ziady, H. H., Alorabi, M., Ayyad, M., \& Sultan, E. A. (2020). Knowledge, perceptions, and attitude of egyptians towards the novel coronavirus disease (COVID-19). Journal of Community Health, 45(5): 881-890. https://doi.org/10.1007/s10900-020-00827-7

Akalu, Y., Ayelign, B., \& Molla, M. D. (2020). Knowledge, attitude and practice towards COVID-19 among chronic disease patients at Addis Zemen Hospital, Northwest Ethiopia. Infection and Drug Resistance, 13(1) : 19491960. https://doi.org/10.2147/IDR.S258736

Alzoubi, H., Alnawaiseh, N., Al-Mnayyis, A., Abu-Lubad, M., Aqel, A., \& Al-Shagahin, H. (2020). Covid-19Knowledge, attitude and practice among medical and non-medical university students in Jordan. J. Pure Appl. Microbiol., 14(1): 17-24. Disponible en: https://pesquisa.bvsalud.org/global-literature-on-novel-coronavirus2019-ncov/resource/pt/covidwho-142234 (Acceso marzo 2021)

Baradaran, A., Ebrahimzadeh, M. H., Baradaran, A., \& Kachooei, A. R. (2020). Prevalence of Comorbidities in COVID-19 Patients: A Systematic Review and Meta-Analysis. Archives of Bone and Joint Surgery, 8(Suppl 1): 247-255. https://doi.org/10.22038/abjs.2020.47754.2346

Beldarraín Chaple, E. R. (2020). La información científica confiable y la COVID- 19. Revista Cubana de Información en Ciencias de la Salud, 31(3), e1609. Disponible en: http://scielo.sld.cu/scielo.php?script=sci_arttext\&pid=S2307-21132020000300004\&lng=es\&tlng=es. (Acceso marzo 2021)

Carro Pérez, E. H., \& Hernández Gómez, A. S. (2016). Percepción de riesgo y prevención en un contexto de emergencia sanitaria: Caso Influenza A (H1N1), 2009. Revista de psicología y ciencias del comportamiento de la Unidad Académica de Ciencias Jurídicas y Sociales, 7(2):20-32. Disponible en: http://www.scielo.org.mx/scielo.php?script=sci_arttext\&pid=S2007-18332016000200003\&lng=es\&tlng=es. (Acceso marzo 2021)

Chan, J. F.-W., Yuan, S., Kok, K.-H., To, K. K.-W., Chu, H., Yang, J., Xing, F., Liu, J., Yip, C. C.-Y., Poon, R. W.-S., Tsoi, H.-W., Lo, S. K.-F., Chan, K.-H., Poon, V. K.-M., Chan, W.-M., Ip, J. D., Cai, J.-P., Cheng, V. C.-C., Chen, H., ... Yuen, K.-Y. (2020). A familial cluster of pneumonia associated with the 2019 novel coronavirus indicating person-to-person transmission: A study of a family cluster. The Lancet, 395(10223): 514-523. https://doi.org/10.1016/S0140-6736(20)30154-9

Chen, M., Zeng, J., Liu, X., Sun, G., Gao, Y., Liao, J., Yu, J., Luo, X., \& Qi, H. (2020). Changes in physiology and immune system during pregnancy and coronavirus infection: A review. European Journal of Obstetrics \& Gynecology and Reproductive Biology, 255(1): 124-128. https://doi.org/10.1016/j.ejogrb.2020.10.035

Chicaíza, L., García Molina, M., \& Urrea-Ríos, I. L. (2021). ¿Economía o salud? Un análisis global de la pandemia de COVID-19. Revista de Economía Institucional, 23(44):171-194. https://doi.org/10.18601/01245996.v23n44.08

Colomé-Hidalgo, M., Herrera Morban, D. A., Méndez Núñez, R., Torres Feliz, Z. L., Méndez Jorge, M., Japa Rodríguez, J., \& Sosa, C. (2021). Conocimiento y percepciones que los adultos tienen del COVID-19, República Dominicana. Ciencia y Salud, 5(1): 27-42. https://doi.org/10.22206/cysa.2021.v5i1.pp27-42

Deng, S.-Q., \& Peng, H.-J. (2020). Characteristics of and Public Health Responses to the Coronavirus Disease 2019 Outbreak in China. Journal of Clinical Medicine, 9(2), 575-65. https://doi.org/10.3390/jcm9020575

Díaz-Rodríguez, Y. L., Vargas-Fernández, M. de L. Á., \& Quintana-López, L. A. (2020). Efectividad de una Intervención educativa sobre el nivel de conocimiento de la COVID-19 en adultos mayores. Universidad Médica Pinareña, 16(3): 570. Disponible en: http://www.revgaleno.sld.cu/index.php/ump/article/view/570 (Acceso marzo 2021)

Falahi, S. \& Kenarkoohi, A. (2021). Diferencias de sexo y género en el resultado de los pacientes con COVID19. Revista de virología médica , 93 (1): 151-152. https://doi.org/10.1002/jmv.26243 
Ferdous, Z., Islam, S., Sikder, T., Mosaddek, A. S., Zegarra-Valdivia, J. A., \& Gozal, D. (2020). Knowledge, attitude, and practice regarding COVID-19 outbreak in Bangladesh: An online-based cross-sectional study. PLOS ONE, 15(10): e0239254. https://doi.org/10.1371/journal.pone.0239254

Galán-Rodas, E., Tarazona-Fernández, A., Palacios-Celi, M., Galán-Rodas, E., Tarazona-Fernández, A., \& PalaciosCeli, M. (2020). Riesgo y muerte de los médicos a 100 días del estado de emergencia por el COVID-19 en Perú. Acta Médica Peruana, 37(2): 119-121. https://doi.org/10.35663/amp.2020.372.1033

García-Saisó, S., Marti, M., Brooks, I., Curioso, W., González, D., Malek, V., Medina, F. M., Radix, C., Otzoy, D., Zacarías, S., dos Santos, E. P., \& D’Agostino, M. (2021). Infodemia en tiempos de COVID-19. Revista Panamericana de Salud Pública, 45(1), e89. https://doi.org/10.26633/RPSP.2021.89

Gil, R., Bitar, P., Deza, C., Dreyse, J., Florenzano, M., Ibarra, C., Jorquera, J., \& Melo, J. (2021). Cuadro clínico del COVID-19. Rev Med Clib Condes, 32(1): 20-29. https://doi.org/10.1016/j.rmclc.2020.11.004

Guzmán, M. E., García, A., Salazar, A., Romero, M., \& Carvajal, A. (2020). Conocimientos de los trabajadores de la salud sobre aspectos generales de la COVID-19. Bol. venez. infectol, 31(2):94-101. Disponible en: https://docs.bvsalud.org/biblioref/2021/06/1252745/02-guzman-m-94-101.pdf (Acceso marzo 2021)

Huynh, G., Nguyen, M. Q., Tran, T. T., Nguyen, V. T., Nguyen, T. V., Do, T. H. T., Nguyen, P. H. N., Phan, T. H. Y., Vu, T. T., \& Nguyen, T. N. H. (2020). Knowledge, attitude, and practices regarding COVID-19 among chronic illness patients at outpatient departments in Ho Chi Minh City, Vietnam. Risk Management and Healthcare Policy, 13(1): 1571-1578. https://doi.org/10.2147/RMHP.S268876

Jin, J.-M., Bai, P., He, W., Wu, F., Liu, X.-F., Han, D.-M., Liu, S., \& Yang, J.-K. (2020). Gender Differences in Patients With COVID-19: Focus on Severity and Mortality. Frontiers in Public Health, 8(152), 1-6. https://doi.org/10.3389/fpubh.2020.00152

Levkovich, I., \& Shinan-Altman, S. (2021). The impact of gender on emotional reactions, perceived susceptibility and perceived knowledge about COVID-19 among the Israeli public. International Health, 1-7. https://doi.org/10.1093/inthealth/ihaa101

Lozano, Y., \& Palacios, E. V. (2021). Factors associated with the hospitalization of COVID-19 patients in a clinic's intensive care unit in 2020. Horiz. méd., 21(1): e1379. http://dx.doi.org/10.24265/horizmed.2021.v21n1.09

Macassi, S. (2020). Comunicación para el cambio de comportamientos y estrategias sanitaria del gobierno peruano frente al COVID-19. Chasqui. Revista Latinoamericana de Comunicación, 1(145):235-258. https://doi.org/10.16921/chasqui.v1i145.4360

Mejia, C. R., Rodriguez-Alarcon, J. F., Carbajal, M., Sifuentes-Rosales, J., Campos-Urbina, A. M., Charri, J. C., GarayRios, L., Al-Kassab-Cordova, A., Mamani-Benito, O., \& Apaza-Tarqui, E. E. (2020). Validación de una escala breve para la medición del nivel de conocimientos básicos acerca del Coronavirus, Perú (KNOW-P-COVID19). Kasmera, 48(1): 1-10. https://doi.org/10.5281/zenodo.3827988

Mungmungpuntipantip, R., \& Wiwanitkit, V. (2021). Medios de comunicación, redes sociales, noticias falsas, información incierta, datos preliminares: Preocupaciones durante la actual pandemia de COVID-19. Revista Peruana de Investigación en Salud, 5(1), 5-6. https://doi.org/10.35839/repis.5.1.913

Qiu, P., Zhou, Y., Wang, F., Wang, H., Zhang, M., Pan, X., Zhao, Q., \& Liu, J. (2020). Clinical characteristics, laboratory outcome characteristics, comorbidities, and complications of related COVID-19 deceased: A systematic review and meta-analysis. Aging Clinical and Experimental Research, 32(9): 1869-1878. https://doi.org/10.1007/s40520-020-01664-3

Qutob, N., \& Awartani, F. (2021). Knowledge, attitudes and practices (KAP) towards COVID-19 among Palestinians during the COVID-19 outbreak: A cross-sectional survey. PLOS ONE, 16(1): e0244925. https://doi.org/10.1371/journal.pone.0244925

Ramírez-Soto, M. C., Arroyo-Hernández, H., \& Ortega-Cáceres, G. (2021). Sex differences in the incidence, mortality, and fatality of COVID-19 in Peru. PLOS ONE, 16(6): e0253193. https://doi.org/10.1371/journal.pone.0253193

Ran, L., Chen, X., Wang, Y., Wu, W., Zhang, L., \& Tan, X. (2020). Risk Factors of Healthcare Workers With Coronavirus Disease 2019: A Retrospective Cohort Study in a Designated Hospital of Wuhan in China. Clinical Infectious Diseases: An Official Publication of the Infectious Diseases Society of America, 71(16): 2218-2221. https://doi.org/10.1093/cid/ciaa287

Raraz Vidal, J. G., Allpas Gomez, H. L., Arenas Lupo, M. R., Raraz Vidal, Y. F., Raraz Vidal, O. B., \& Gonzales Rengifo, G. (2021). Conocimiento de signos de alarmas y síntomas de la enfermedad de COVID-19 en la 
población de Lima, Perú. Revista Cubana de Investigaciones Biomédicas, 40(2): e1085. Dispomible en: http://www.revibiomedica.sld.cu/index.php/ibi/article/view/1085/1023 (Acceso marzo 2021)

Rivera Diaz, P. A., Rubiano Daza, H., Quintero Quimbaya, J. C., Hoyos Armero, D. P., Herrera Ramírez, C., Rivera Ospitia, S. M., \& Ortiz, C. P. (2020). Conocimiento preventivo y su práctica entre la población de Colombia hacia la enfermedad por Coronavirus (COVID-19): Una perspectiva de género. Rev. colomb. ciencias quim. farm, 49(3): 776-789. https://doi.org/10.15446/rcciquifa.v49n3.91344

Roth, C., Breckner, A., Moellinger, S., Schwill, S., Peters-Klimm, F., Szecsenyi, J., Stengel, S., \& Wensing, M. (2021). Beliefs and practices among primary care physicians during the first wave of the COVID-19 pandemic in Baden-Wuerttemberg (Germany): An observational study. BMC Family Practice, 22(1): 86. https://doi.org/10.1186/s12875-021-01433-9

Ruiz Aquino, M., Díaz Lazo, A. V., Ubillús, M., Aguí Ortiz, A. K., \& Bravo, V. (2021). Percepción de conocimientos y actitudes frente a COVID-19 en un grupo de ciudadanos de la zona urbana de Huánuco. Revista de la Facultad de Medicina Humana, 21(2): 292-300. https://doi.org/10.25176/rfmh.v21i1.3352

Sánchez Ortiz, M. P. (2016). Efectividad de tres intervenciones para mejorar la adherencia al cribado del cáncer de cérvix. Tesis Doctoral, Universidad de Castilla-La Mancha. https://ruidera.uclm.es/xmlui/handle/10578/9131

Sohrabi, C., Alsafi, Z., O’Neill, N., Khan, M., Kerwan, A., Al-Jabir, A., Iosifidis, C., \& Agha, R. (2020). World Health Organization declares global emergency: A review of the 2019 novel coronavirus (COVID-19). International Journal of Surgery, 76(1): 71-76. https://doi.org/10.1016/j.ijsu.2020.02.034

Struyf, T., Deeks, J. J., Dinnes, J., Takwoingi, Y., Davenport, C., Leeflang, M. M., Spijker, R., Hooft, L., Emperador, D., Domen, J., Horn, S. R. A., Bruel, A. V. den, \& Group, C. C.-19 D. T. A. (2021). Signs and symptoms to determine if a patient presenting in primary care or hospital outpatient settings has COVID-19. Cochrane Database of Systematic Reviews, 2021(2): 1-207. https://doi.org/10.1002/14651858.CD013665.pub2

Ticona Huaroto, C., Espinoza Silva, M. M., Ticona Chávez, E., Ticona Huaroto, C., Espinoza Silva, M. M., \& Ticona Chávez, E. (2020). COVID-19. El manejo ambulatorio durante la transmisión comunitaria intensa. Anales de la Facultad de Medicina, 81(2): 211-217. https://doi.org/10.15381/anales.v81i2.18406

Vázquez Chacón, M., Cabrejos Llontop, S., Yrigoin Pérez, Y., Robles Alfaro, R., \& Toro Huamanchumo, C. J. (2019). Adicción a internet y calidad de sueño en estudiantes de medicina de una Universidad peruana, 2016. Revista Habanera de Ciencias Médicas, 18(5): 817-830. Disponible en: http://scielo.sld.cu/scielo.php?script=sci_arttext\&pid=S1729-519X2019000500817\&lng=es. Epub 06-Oct2019 (Acceso marzo 2021)

Wu, J., Liu, J., Zhao, X., Liu, C., Wang, W., Wang, D., Xu, W., Zhang, C., Yu, J., Jiang, B., Cao, H., \& Li, L. (2020 a). Clinical Characteristics of Imported Cases of Coronavirus Disease 2019 (COVID-19) in Jiangsu Province: A Multicenter Descriptive Study. Clinical Infectious Diseases: An Official Publication of the Infectious Diseases Society of America, 71(15): 706-712. https://doi.org/10.1093/cid/ciaa199

Wu, C., Chen, X., Cai, Y., Xia, J., Zhou, X., Xu, S., Huang, H., Zhang, L., Zhou, X., Du, C., Zhang, Y., Song, J., Wang, S., Chao, Y., Yang, Z., Xu, J., Zhou, X., Chen, D., Xiong, W., ... Song, Y. (2020 b). Risk Factors Associated With Acute Respiratory Distress Syndrome and Death in Patients With Coronavirus Disease 2019 Pneumonia in Wuhan, China. JAMA Internal Medicine, 180(7): 934-943. https://doi.org/10.1001/jamainternmed.2020.0994

Zhou, F., Yu, T., Du, R., Fan, G., Liu, Y., Liu, Z., Xiang, J., Wang, Y., Song, B., Gu, X., Guan, L., Wei, Y., Li, H., Wu, X., Xu, J., Tu, S., Zhang, Y., Chen, H., \& Cao, B. (2020). Clinical course and risk factors for mortality of adult inpatients with COVID-19 in Wuhan, China: A retrospective cohort study. The Lancet, 395(10229): 10541062. https://doi.org/10.1016/S0140-6736(20)30566-3

Zhu, N., Zhang, D., Wang, W., Li, X., Yang, B., Song, J., Zhao, X., Huang, B., Shi, W., Lu, R., Niu, P., Zhan, F., Ma, X., Wang, D., Xu, W., Wu, G., Gao, G. F., \& Tan, W. (2020). A Novel Coronavirus from Patients with Pneumonia in China, 2019. New England Journal of Medicine, 382(8): 727-733. https://doi.org/10.1056/NEJMoa2001017 